\title{
Role of Herpes Virus in Periodontal Diseases
}

\author{
Dr. Sheenu Varghese
}

\begin{abstract}
Periodontitis is a chronic inflammatory disease with acomplex aetiopathogenesis . Oral Biofilm has a primary role in the development of periodontitis. Microbial biofilm constitutes a multiple array of bacteria, viruses, fungi well present within periodontal tissue. Oral biofilm present in periodontal pocket causes tissue destruction. The role of viruses proves a new paradigm in explaining the link pathway and periodontal destruction. This review explains the role of viruses in pathogenesis of periodontitis and its implication in disease progression and therapy.
\end{abstract}

\section{Introduction}

Periodontitis is a multifactorial, chronic disease that progresses by destruction of supporting structures of teeth like cementum, alveolar bone, periodontal ligament(1). The main cause of periodontitis is the oral biofilm, with multiple microorganisms present such as bacteria and viruses. Whether the association between viruses and bacteria are causal in periodontitis, is still a dilemma.

\section{Viruses In Periodontitis}

Viruses are obligate intracellular organisms, which are pathogenically and metabolically inert outside the host cell. Classification of viruses is based on type of nucleic acid genome(DNA or RNA) present(2).Among the viruses present, Herpes Simplex virus can be classified into: Herpessimplex virus type 1(HSV1),type 2(HSV2), varicella zoster(VZV), human cytomegalovirus(HCMV), human herpes virus 6(HHV6), human herpes virus 7(HHV7), Epstein barr virus(EBV)and human herpes virus 8(HHV-8). HHV-6 and HHV -8 were detected in biopsies from periodontal lesions. (3)

Periodontopathic bacteria, such as Porphyromonas gingivalis, possess virulence factors involved in neutralizing local host defences, colonized periodontal sites and destroying periodontal tissues.(4). Healthy periodontal sites harbour microbiota of predominantly gram positive facultative bacteria, where as periodontal lesions contain a large variety of gram negative anaerobic species(5). Viruses are known to infect inflammatory cells of the periodontium, they are present more frequently in diseased sites than in healthy sites.

According to APP classification of Periodontitis, Periodontitis can be classified into Chronic periodontitis and Aggresive periodontitis. In Aggressive Periodontitis, Cytomegalovirus and Porphyromonas gingivalis were detected in sites with localised aggressive periodontitis in Afro-caribbean adolescents.(6) Epstein barr viruses are commonly seen with less involvement of HSV1 virus seen only in advanced sites of generalised aggressive periodontitis.(7). In Chronic periodontitis CMV,EBV1, HSV1 are the main herpes viruses seen(8). The latent form of CMV is seen in the majority of chronic periodontitis sites, which might account for the slow progression of the disease (9). CMV and HHV7 are common in periodontitis affected sites and less common in healthy sites (10). A wide variation in the occurrence of HSV (13-100\%), EBV(3-89\%) and $\mathrm{CMV}(0.3-83 \%)$ have been reported in periodontitis lesions due to multiple factors involved(11). In adults, chronic periodontitis, the presence of subgingival HCMV or EBV-1 DNA is related to an elevated occurrence of periodontal pathogens like P. gingivalis(12).

\section{Pathogenesis:}

Pathogenic process of periodontitis may be as a result of direct cause of various virus infections or damage caused due to the host defence. Despite a long study and research in pathogenesis of periodontitis, there still remains a question about its probable cause.

Herpes virus can alter structural cells and host defence cell of periodontium which will reduce the host resistance against multiplication of periodontal pathogens and subgingival colonisation $(3,13)$ Herpes virus may cause direct cytopathic effects on inflammatory cells like leukocytes, lymphocytes, macrophages and on fibroblasts, keratinocytes and endothelial cells(14).

HCMV and EBV 1 can infect or alter functions of monocytes, macrophages and lymphocytes. (15). Herpes virus infection may increase the pathogenicity of periodontal microbiota. Proteins which are expressed by herpesvirus on eukaryotic cell membrane may act as new bacterial binding sites (16). Polymorphonuclear leukocytes which are cells for controlling periodontopathic bacteria, herpes virus may induce abnormalities in its adherence, chemotaxis, phagocytic and bacterial activities (17). 
Herpes viral infection shows altered inflammatory mediators and cytokines responses .HCMV infection can up regulate interleukin $1 \beta$ and tumour necrosis factor $\alpha$ (TNF- $\alpha$ ). EBV infection of lymphocytes shows a shift in lymphocytes counts towards predominance of B lymphocytes Iplasma cells. These cells are prominent in progressive periodontal lesions (18).

HCMV and HSV can induce cell mediated immuno suppression by reducing the cell surface expression of MHC CLASS 1 molecule, which will thereby interfere with T lymohocytes recognition . HCMV can suppress antigen specific cytotoxic T lymphocytes functions, resulting in decrease in circulatory CD4+ cells and increase in CD8 suppressor cells which lead to impairment of cell mediated immunity. Acute EBV infection and infectious mononucleosis can induce polyclonal B lymphocytes activation with generation of neutropenia and anti neutrophil antibodies. EBV infected B lymphocytes may shed viral structural antigens that result in production of blocking antibodies, immune complex function and T suppressor cell activation. (19)

\section{Viral Diagostic Methods}

There are different diagnostic methods to identify viruses in perodontitis. Initially identification of virus has traditionally been based on cell culture to detect characteristics cytopathic effects ,morphologic determination of intracytoplasmic to identify viral antigens in clinical specimens.(20)The presence of herpesvirus in periodontium is also confirmed using flow cytometry, DNA probes and immunofluorescene staining.

Other popular technique is Polymerase chain reaction which is becoming the standard for identification and quantification of periodontal herpesvirus (21). Several types PCRs are used like Nested PCR, real time PCR, multiple PCR. In nested PCR it shows more periodontal sites that are positive for HCMV than viral culture or real time PCR (22). Nested PCR technique is more efficient in detecting low viral loads. (23) In multiple PCRs , multiple organisms can be detected.PCR based studies of periodontal herpesvirus have targeted different genomic regions and used to extract the target nucleic acid with different efficiency .Negative PCR may occur because of the absence of virus at the time of periodontal sampling(24).Ultrasensitive PCR techniques help to identify herpesvirus in unhealthy control sites. Periodontitis patients with healthy periodontal sites will have more herpesvirus than patient of healthy periodontal sites with healthy periodontium.(25)EBV and HCMV have been identified using a variety of PCR primers in platforms of end point detection PCR, nested PCR, real time and reverse transcription PCR.(26)

\section{Herpesvirus Bacterial Interactions In Periodontitis}

The interaction between herpesviruses and bacteria can be bidirectional with bacterial enzymes or inflammation induced factors, having the potential to activate periodontal herpesvirus.(16) Periodontitis has a sequential infectious process that proceeds from bacteria to herpesvirus to bacteria .(14) Initially bacteria present in the dental biofilm induce gingivitis which then permits latent herpesvirus which is embedded in the DNA of macrophages, T lymphocytes and B lymphocytes to infiltrate the periodontium(27). Reactivation of the latent herpesvirus can occur spontaneously or during the period of decreased host defence seen because or drug induced immunosuppression, emotional stress, concurrent infection, hormonal changes, physical trauma. (28) Herpes virus activation leads to increased inflammatory mediator response triggering a cytokine or chemokine of $1 \mathrm{~L}-1 \beta, \mathrm{TNF} \alpha, 1 \mathrm{~L} 6$, prostaglandins, interferons, and other mediators which have potential to propagate bone resorption (29).Most of the herpes virus, associated cytokines and chemokines are prominent in periodontal lesions(30). In avicious circle, the triggering of cytokine responses may activate latent herpes virus which will further aggravate periodontal disease. Moreover, most immune competent individuals experience episodes of oral herpes virus reactivation, but it will last only for few hours or day, which is very less time span to initiate periodontal disease. (31)

Association between systemic diseases like cardiac diseases and viruses is still a question. Vascular disease occur at a higher frequency as a result of combined infections with herpes simplex virus and Chlamydia pneumonia.(32) HSV and periodontopathic bacteria in aggregate and herpesvirus 8 have also been associated with vascular diseases.(33)

\section{Therapeutic Implications}

Many therapeutic methods has been implicated in recent years to eradicate the infection caused by herpesvirus in periodontium. Conventional periodontal therapy can reduce the periodontal load of herpesviruses. Mechanical debridement has also showed suppressed sub gingival EBV(16). The orally administered acyclovir prodrug, valacyclovir can reach serum concentration same to those of intravenously administered acyclovir and is used for a variety of herpes viral diseases. (34) Except in immunosuppressed individuals, prolong treatment with valacyclovir at dosage of 500-1000mgldaily is well tolerated.( 35 )Still studies are going on to detect whether antiviral drugs are effective in treatment of herpes virus or other viruses in periodontitis. 


\section{Conculsion:}

Prevention and elimination of periodontal disease is associated with the complete elimination of periodontopathic bacteria and viruses from the oral environment. An important question is whether the herpesvirus - periodontal association is based etiologically or is just an epiphenenoem to gingival inflammation. Periodontopathogenic bacteria, EBV and HCMV seemed to act well and result in increased risk for the occurrence and spread of periodontitis. Coinfection of active herpesvirus and periodontitis bacteria may constitute major causes of periodontitis.A good understanding of the cause of periodontitis is important to develop clinically relevant therapies, so as to ensure long lasting disease control from viruses. Control of herpesvirus with vaccination maybe the future for prevention of periodontitis with diminishing role for traditional periodontal therapy of surgery and antibiotics. With further and future researches of virus in periodontitis can lead to progress in prevention and treatment of periodontal disesases.

\section{References:}

[1] Paster BJ, Dewwhrist FE. Molecular microbial diagnosis. Periodontontol 2000 2009: 51: 38-44

[2] Jarvis M A, Nelson J A. Mechanics of human cytomegalovirus persistence and latency .Front Biosci $2002 ; 35$ :3-16

[3] Contreas A, Nowzari H, Slots J. Herpesviruses in periodontal pocket and gingival tissue specimens, Oral Microbiol Immunol 2000: 15: $15-18$

[4] Holt S, Ebersole JL. Porphyromonas gingivalis, Treponema denticola and Tannerella forsythia: The red complex ,a prototype polybacterial pathogenic consortium in periodontitis. Periodontol 2000 2005: 38 :72-122

[5] Slots J, subgingival microflora and periodontal disease. J Clin Periodontal 1979: 6:351-382

[6] Michalowicz BS, Ronderos M, Camara-silva R, Contreas A, Slots J. Human herpesvirus and porphyromonas gingivalis are associated with early onset periodontitis. J Periodontol 2000: 71 :981-988

[7] Skrepcinski FB, Tetrevs, Rams TE, Sutton B, Contreas A Slots J.; Periodontal disease in hopi native American teenagers .J Dent res 199: $76: 439$.

[8] Hochman N, Zakay rones Z, Shohat $\mathrm{H}$ et al. Antibodies to cytomeglo and Epstein barr viruses in human saliva and gingival fluid $1998: 21: 131-139$

[9] Wu YM, Yan J, Ojcius DM, Chen LL. Correlation between infection with different genotypes of HCMV and EBV in subgingival samples and periodontal status of patients 2007:45:3665-3670

[10] Thomasini RL, Bonon SH, Durante P, Costa SC. Correlation of cytomegalovirus and human herpesvirus 7 with CD3+CD4+cells in chronic periodontitis, J Periodontal res2012; 47 ;114-20

[11] Kubar A, Saygun I, Yapar M, Slots J. Real time polymerase chain reaction quantification of human cytomegalovirus and Epstein barr virus in periodontal pocket and adjacent gingival of periodontitis lesions J. Periodontol res 2005: 40; 97-104

[12] Slots J, Kamma J J, Sugar C. The herpes virus-porphyromonas gingivalis-periodontitis axis. J Periodontol res 2003.;38:318

[13] Cassai E, Galvan M, Trombelli L, Rotola A.HHV-6,HHV-7, HHV-8 in gingival biopsies from chronic adult periodontitis patients. J CLIN Periodontol 2003: 30 :184-191

[14] Slots J, Contreas A. Herpesvirus: A unifying causative factor in periodontitis? Oral microbial immunol 2000;15;276-9

[15] Drew W L.Laboratory diagnosis of infectious diseases 1988)

[16] Slots J .Herpesviral bacterial interaction in periodontal disease. Periodontol 2000 2010: 52 :117-140

[17] Slots J, Saygun I, Sabeti M Kubar A.Epstein barr virus in oral diseases.J Periodont res $2006: 41$ :235-244

[18] A Slots J Herpesviruses in periodontal disease. Periodontol 2000 2005: 38 :33-62

[19] Slots J Human viruses in periodontitis. Periodontol 2000 2010; 53; 89-110

[20] Babita R, Pawar, Avneesh H , Tejnani, Pramod P.Maarwar, Ameet M.Mani:Herpes virus;A key missing piece of the periodontopathogenic jigsaw puzzle

[21] Kubar A, Sayguni I, Yapar M O,Slots J, Real Time PCR quantification of cytomegalovirus in aggressive periodontitis lesion using taqman technique J Periodontol res 2004.

[22] Botero J E, Vidal C, Contreras A, Paara B. Comparsion of nested PCR, real TIME PCR and viral culture for the detection of cytomegalovirus in subgingival samples. Oral microbial immunol 2008: 23:239-244

[23] Rotola A, Cassia E,Farina R, Caselli E, Gentili V.Human herpesvirus 7,Epstein barr virus and human cytomegalovirus in periodontal tissues of periodontally diseased and healthy subjects. J Clin periodontal 2008:35:831-837

[24] Caliendo AM, Shahbazin MD et al. A commtable cytomegalovirus calibrator is required to improve the agreement of viral load values between laboratories.Clin Chem 2009: 55: 1701-1710

[25] Dawson DR, Wang C, Jacob RJ, Mille CS et al. Salivary levels of Epstein barr virus DNA correlate with subgingival levels, not severity of periodontitis Oral dis 2009: 15: 554-559

[26] Combs DR, Reiley, Dawson DR III,Miller CS et al. Detection of human cytomegalovirus in dental plaque from individual periodontal sites by real time polymerase chain reaction. Oral Surg Oral Med 2008: $106: 840-844$

[27] Contreras A, Zadeh, Nowzari H, Slots J Herpesvirus infection of inflammatory cells in human periodontitis. Oral microbial immunol 1994:14:206.

[28] Reddy MS Reaching a better understanding of non disease and the implication of periodontal infections. Periodontol 2000 2007: 44 : $9-14$

[29] Kwashima N, Stanshenko P. Expression of bone resorptive and regulatory cytokines in murine periapical inflammation .Arch oral boil 1999: 44: 55-66

[30] Graves DT, Cochran D. The contribution of interleukin 1 and tumour necrosis factor to periodontal tissue destruction. J Periodontol 2003:74:391-401

[31] Mark KE, Wald, Selke S, Olin 1, Corey 1. Rapidly cleared episodes of herpes simplex virus reactivation in immunocompetent adults J Infect dis 2008:198:1141-1149

[32] Roivainen M, Viik et al. Infectionsand inflammation and risk of coronary heart disease.Circulation $2000: 101: 252-257$

[33] Vercelloti GM. Overview of infections and cardiovascular diseases. J Allergy Clin immunol 2001; 108;117-120

[34] Patel R. Valaciclovir :Development, clinical utility and potential. Expert opin investing 1997:6:16-21

[35] Bakaletz LO. Viral potentiation of bacterial superinfection of the respiratory tract. Trends microbial $1995: 3: 110-4$ 